VOLUME 30 (2021) 285-303

DOI: $10.24330 /$ ieja.969940

\title{
LATTICE DECOMPOSITION OF MODULES
}

\author{
J. M. García, P. Jara and L. M. Merino \\ Received: 14 September 2020; Revised: 27 December 2020; Accepted 12 January 2021 \\ Communicated by Abdullah Harmanc1
}

\begin{abstract}
The first aim of this work is to characterize when the lattice of all submodules of a module is a direct product of two lattices. In particular, which decompositions of a module $M$ produce these decompositions: the lattice decompositions. In a first étage this can be done using endomorphisms of $M$, which produce a decomposition of the $\operatorname{ring} \operatorname{End}_{R}(M)$ as a product of rings, i.e., they are central idempotent endomorphisms. But since not every central idempotent endomorphism produces a lattice decomposition, the classical theory is not of application. In a second step we characterize when a particular module $M$ has a lattice decomposition; this can be done, in the commutative case in a simple way using the $\operatorname{support,} \operatorname{Supp}(M)$, of $M$; but, in general, it is not so easy. Once we know when a module decomposes, we look for characterizing its decompositions. We show that a good framework for this study, and its generalizations, could be provided by the category $\sigma[M]$, the smallest Grothendieck subcategory of Mod $-R$ containing $M$.
\end{abstract}

Mathematics Subject Classification (2020): 16D70, 18E35, 13C60

Keywords: Module, ring, lattice, lattice decomposition, Grothendieck category

\section{Introduction}

Let $M$ be a (unitary) right $R$-module over a (unitary) ring $R$; it is well known that decompositions of $M$, as a direct sum of two submodules, are parameterized by idempotent endomorphisms in $S=\operatorname{End}_{R}(M)$. Thus, if $M=N \oplus H$, there exists $e \in \operatorname{End}_{R}(M)$ such that $N=e(M)$, and $H=(1-e)(M)$. In general, $e$ is not necessarily central in $S$, hence it does not produce a decomposition of $S$ in a direct product of two rings. In this paper we deal with some special decompositions of modules so that the lattice $\mathcal{L}(M)$, of all submodules of $M$, will be a direct product of two lattices: the lattice decomposition of $M$.

This kind of decompositions are of interest as if $M=N \oplus H$ is a lattice decomposition, then every submodule $X \subseteq M$ can be expressed as a direct sum, $X=(X \cap N) \oplus(X \cap H)$, and this property has great importance in order to study the structure of $M$. 
From this point of view, we first recall that a lattice decomposition defines an element $N \in \mathcal{L}(M)$ which is distributive; this means that for any $X, Y \in \mathcal{L}(M)$, the sublattice of $\mathcal{L}(M)$ generated by $N, X$ and $Y$ is distributive: a well known notion in lattice theory; in addition, it is complemented, as $M=N \oplus H$. Elements of this kind have good properties as members of the lattice $\mathcal{L}(M)$.

We exploit the existence of complemented distributive submodules of a right $R-$ module $M$. In order to characterize them first we deal with endomorphisms. Thus we show a characterization of those central idempotent endomorphisms in $\operatorname{End}_{R}(M)$ that define lattice decompositions of $M$. In the particular case of modules over a commutative ring, these idempotent endomorphisms are those that belong to the closure of $R$ in $\operatorname{End}_{R}(M)$, with respect to the finite topology. In addition, we show that every complemented distributive submodule $N \subseteq M$ is stable under any endomorphism $f \in \operatorname{End}_{R}(M)$.

The behavior of complemented distributive submodules is also studied, thus it is shown that for any complemented submodule $N \subseteq M$, and any index set $I$ we get a complemented distributive submodule $N^{(I)} \subseteq M^{(I)}$. This property, together with the known characterization of distributive submodules as those submodules $N \subseteq M$ such that for every submodule $H \subseteq M$ the factor modules $N /(N \cap H)$ and $H /(N \cap H)$ have no non-zero isomorphic subfactors allow us to extend the theory to categories which are defined directly from $M$, as the category $\sigma[M]$. Indeed, we recover a decomposition theory for these categories showing that there exists a closed relationship between decomposition of $\sigma[M]$, as a product of two subcategories, and lattice decompositions of $M$ as a right $R$-module.

The paper is organized in sections. In the second section we recall the notions of product of lattices and the consequences of the existence of a lattice decomposition. In sections three and four we study distributive submodules of a right $R$-module, and show that simple subfactors are decisive to characterize complemented distributive submodules. In particular, if the base ring $R$ is commutative, a direct sum decomposition $M=N \oplus H$ is a lattice decomposition if, and only if, $\operatorname{Supp}(N) \cap \operatorname{Supp}(H)=\varnothing$. One of the main aims is to relate complemented distributive submodules $N \subseteq M$ and central idempotent endomorphisms. Thus we show, in examples, that not every such idempotent endomorphism defines a distributive submodule, and in Theorem (4.11) we show that it is necessary and sufficient that this endomorphism stabilizes every submodule. In consequence, these idempotent endomorphisms are close to multiplication by elements of $R$, and in Proposition (4.15) we show that they must belong to the closure of $R$ in $\operatorname{End}_{R}(M)$ with respect to the 
finite topology, whenever $R$ is commutative. In order to extend these results to categories, in section five we establish, Proposition (5.1), showing that direct powers preserve complemented distributive submodules. The strong relationship between decompositions of the category $\sigma[M]$ and lattice decompositions of the module $M$ is also studied.

References to undefined terms can be find either in the following papers: [6], [7] and [10], or in the books: [5], [8] and [12].

\section{Product of lattices}

Let $L_{1}, L_{2}$ be lattices, and define in the cartesian product $L_{1} \times L_{2}$ the operations:

$$
\begin{aligned}
& \left(a_{1}, a_{2}\right) \wedge\left(b_{1}, b_{2}\right)=\left(a_{1} \wedge b_{1}, a_{2} \wedge b_{2}\right) \text { and } \\
& \left(a_{1}, a_{2}\right) \vee\left(b_{1}, b_{2}\right)=\left(a_{1} \vee b_{1}, a_{2} \vee b_{2}\right),
\end{aligned}
$$

then $\left(L_{1} \times L_{2}, \wedge, \vee\right)$ is a lattice, and the canonical projections $p_{i}: L_{1} \times L_{2} \longrightarrow L_{i}$, $i=1,2$, are lattice maps. In addition, $\left(L_{1}, \times L_{2},\left\{p_{1}, p_{2}\right\}\right)$ is the product of $L_{1}$ and $L_{2}$ in the category of lattices and lattice maps.

Examples of lattices appear in many different contexts; we are interested in those lattices that appear in module theory, i.e., if $R$ is a (unitary) ring and $M$ a right (unitary) $R$-module, in the lattice $\mathcal{L}(M)$ of all submodules of $M$, and in the particular problem of characterizing when $\mathcal{L}(M)$ is the product of two lattices.

For any right $R$-module $M$ the lattice $\mathcal{L}(M)$ has extra properties in addition to those that define a lattice, for instance:

(1) $\mathcal{L}(M)$ is bounded, i.e., there exists a bottom element, $0 \subseteq M$, and a top one, $M$.

(2) $\mathcal{L}(M)$ is modular, i.e., for any $N_{1}, N_{2}, N_{3} \subseteq M$ such that $N_{1} \subseteq N_{3}$, we have $\left(N_{1}+N_{2}\right) \cap N_{3}=N_{1}+\left(N_{2} \cap N_{3}\right)$.

If $\mathcal{L}(M)$ is a product of lattices, using that $\mathcal{L}(M)$ is bounded, the following easy results holds.

Lemma 2.1. If $\mathcal{L}(M)$ is the product of two lattices, say $\mathcal{L}(M)=\mathcal{L}_{1} \times \mathcal{L}_{2}$, with projections $\left\{p_{1}, p_{2}\right\}, M=\left(M_{1}, M_{2}\right)$, and $0=\left(0_{1}, 0_{2}\right)$, then $\mathcal{L}_{1}$ satisfies the following properties:

(1) $\mathcal{L}_{1}$ is a bounded lattice with bottom $0_{1}$ and top $M_{1}$.

(2) The map $q_{1}: \mathcal{L}_{1} \longrightarrow \mathcal{L}(M)$, defined $q_{1}(X)=\left(X, 0_{2}\right)$, is a one-to-one lattice map.

(3) There is a lattice isomorphism between $\mathcal{L}_{1}$ and $\left\{H \subseteq M \mid H \subseteq M_{1}\right\}$. 
(4) The map $h_{1}: \mathcal{L}_{1} \longrightarrow \mathcal{L}(M)$, defined $h_{1}(X)=\left(X, M_{2}\right)$, is a one-to-one lattice map.

(5) There is a lattice isomorphism between $\mathcal{L}_{1}$ and $\left\{L \subseteq M \mid M_{2} \subseteq L\right\}$.

The same properties hold for the lattice $\mathcal{L}_{2}$. In particular, $M$ is the direct product of $M_{1}$ and $M_{2}$, and there is a lattice isomorphism $\mathcal{L}(M) \cong \mathcal{L}\left(M_{1}\right) \times \mathcal{L}\left(M_{2}\right)$.

Proof. (1) For any $X \in \mathcal{L}_{1}$ we have $\left(0_{1}, 0_{2}\right)=0 \leq\left(X, 0_{2}\right)$, hence $0_{1}=p_{1}\left(0_{1}, 0_{2}\right) \leq$ $p_{1}\left(X, 0_{2}\right)=X$, and $\left(X, M_{2}\right) \leq M=\left(M_{1}, M_{2}\right)$; therefore, $X=p_{1}\left(X, M_{2}\right) \leq$ $p_{1}\left(M_{1}, M_{2}\right)=M_{1}$.

(2) and (3) It is clear that $q_{1}$ is a lattice map, and the announced isomorphism is given by $q_{1}$.

(4) and (5) It is clear that $h_{1}$ is a lattice map, and the announced isomorphism is given by $h_{1}$.

Observe that $M_{1} \cap M_{2}=0$ and $M_{1}+M_{2}=M$, hence $M=M_{1} \times M_{2}$.

A right $R$-module $M$ has a lattice decomposition whenever $\mathcal{L}(M)$ is a product of two nontrivial lattices.

It is clear that not every decomposition of a module $M$ as a direct product gives a lattice decomposition of $\mathcal{L}(M)$ in a product of lattices. See the following example.

Example 2.2. Consider the abelian group $M=\mathbb{Z}_{2} \times \mathbb{Z}_{2}$, the lattice of subgroups of $M$ is not a product of two nontrivial lattices; in particular, $\mathcal{L}(M)$ is not the product $\mathcal{L}\left(\mathbb{Z}_{2}\right) \times \mathcal{L}\left(\mathbb{Z}_{2}\right)$.

Example 2.3. A commutative ring $A$ has a lattice decomposition if, and only if, $A$ is the product of two nontrivial ideals. Indeed, if $\mathcal{L}(A)=\mathcal{L}_{1} \times \mathcal{L}_{2}$, there exist ideals $\mathfrak{a}_{1}, \mathfrak{a}_{2} \subseteq A$ such that $A=\mathfrak{a}_{1} \times \mathfrak{a}_{2}$. Otherwise, if $A=\mathfrak{a}_{1} \times \mathfrak{a}_{2}$, there are idempotent elements $a_{i} \in \mathfrak{a}_{i}, i=1,2$, such that $1=a_{1}+a_{2}$. For any ideal $\mathfrak{a} \subseteq A$ we have $\mathfrak{a}=\mathfrak{a} a_{1} \times \mathfrak{a} a_{2}$, and an isomorphism $\mathcal{L}(A) \cong \mathcal{L}\left(\mathfrak{a}_{1}\right) \times \mathcal{L}\left(\mathfrak{a}_{2}\right)$.

Example 2.4. This result for non-commutative rings does not hold. Let us consider a field $K$ and the matrix ring $M_{2}(K)$ of all square matrices of order 2. The ideals $\mathfrak{a}_{1}=\left(\begin{array}{cc}K & K \\ 0 & 0\end{array}\right)$ and $\mathfrak{a}_{2}=\left(\begin{array}{cc}0 & 0 \\ K & K\end{array}\right)$ satisfy $M_{2}(K)=\mathfrak{a}_{1} \oplus \mathfrak{a}_{2}$. Otherwise, each $\mathfrak{a}_{i}$ is a simple right $M_{2}(K)$-module, hence $\mathcal{L}\left(\mathfrak{a}_{i}\right)=\left\{0, \mathfrak{a}_{i}\right\}$, but $\mathcal{L}\left(M_{2}(K)\right)$ is not the product $\mathcal{L}\left(\mathfrak{a}_{1}\right) \times \mathcal{L}\left(\mathfrak{a}_{2}\right)$ because, for any $0 \neq a \in K$, the right ideal $\left(\begin{array}{ll}1 & 0 \\ a & 0\end{array}\right) M_{2}(K)$ is not in this product. See Corollary (4.12) to determine when a ring $R$ have a lattice decomposition as right $R$-module. 
Our aim in the next section shall be to show some characterizations of modules having a lattice decomposition.

\section{Distributive submodules}

Let $M$ be a right $R$-module. If $N \subseteq M$ is a submodule, there exists a short exact sequence $0 \rightarrow N \rightarrow M \rightarrow M / N \rightarrow 0$, and maps

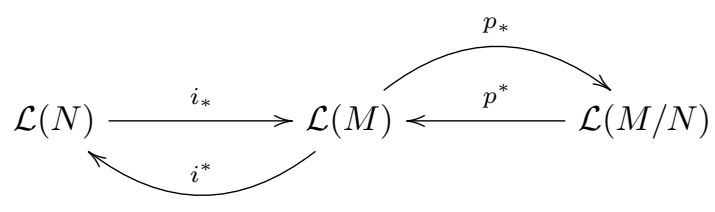

Defined by:

(1) $i_{*}(X)=X$, for every $X \subseteq N$; it is a lattice homomorphism.

(2) $i^{*}(Y)=Y \cap N$, for every $Y \subseteq M$; it satisfies $i^{*}\left(Y_{1} \wedge Y_{2}\right)=i^{*}\left(Y_{1}\right) \wedge i^{*}\left(Y_{2}\right)$, but it is not a lattice homomorphism unless $N$ satisfies $\left(Y_{1}+Y_{2}\right) \cap N=\left(Y_{1} \cap N\right)+\left(Y_{2} \cap N\right)$ for any $Y_{1}, Y_{2} \subseteq M$.

(3) $p_{*}(Y)=(Y+N) / N$, for every $Y \subseteq M$; it satisfies $p_{*}\left(Y_{1} \vee Y_{2}\right)=p_{*}\left(Y_{1}\right) \vee p_{*}\left(Y_{2}\right)$, but it is not a lattice homomorphism unless $N$ satisfies $\left(Y_{1} \cap Y_{2}\right)+N=\left(Y_{1}+\right.$ $N) \cap\left(Y_{2}+N\right)$ for any $Y_{1}, Y_{2} \subseteq M$.

(4) $p^{*}(Y / N)=Y$, for every $Y / N \subseteq M / N$; it is a lattice homomorphism.

Thus, in the above diagram all maps are lattice maps if, and only if, $N$ satisfies conditions in (2) and in (3). In [5] an element in a lattice satisfying property in (3) is called a distributive element, and if it satisfies property in (2), a dual distributive element, proving in [5, Theorem III.2.6] that an element in a modular lattice is distributive if and only if it is dual distributive if, and only if, the sublattice generated by $N, Y_{1}$ and $Y_{2}$, in the former notation, is distributive.

We call a submodule $N \subseteq M$ distributive whenever $\left(Y_{1} \cap Y_{2}\right)+N=\left(Y_{1}+N\right) \cap$ $\left(Y_{2}+N\right)$ for any $Y_{1}, Y_{2} \subseteq M$, or equivalently if $\left(Y_{1}+Y_{2}\right) \cap N=\left(Y_{1} \cap N\right)+\left(Y_{2} \cap N\right)$ for any $Y_{1}, Y_{2} \subseteq M$, and observe that this is also equivalent to the condition that the sublattice of $\mathcal{L}(M)$, generated by $N, Y_{1}$ and $Y_{2}$, is distributive.

Our aim in this section is to characterize distributive submodules of a module. To do that we need the following definition. Let $M$ be a right $R$-module, a subfactor of $M$ is a submodule of a homomorphic image of $M$. Observe that for any subfactor $L$ of a right $R$-module $M$, and any submodule $K \subseteq L$ we may build a commutative 
diagram

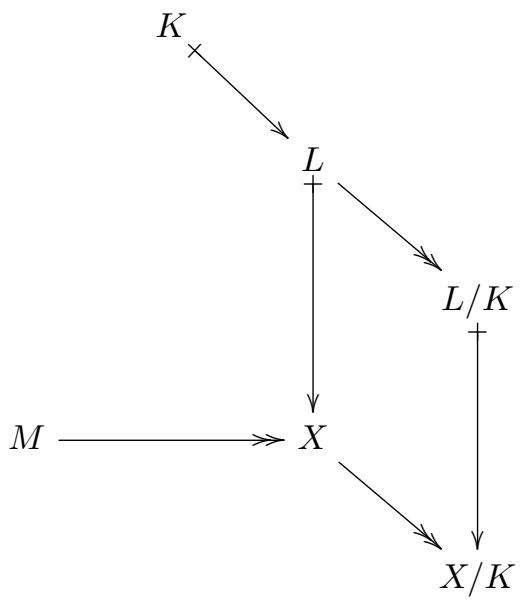

Therefore, if $L$ is a subfactor of $M$, then $K$ and $L / K$ are also subfactors of $M$.

This situation can be enhanced if we make use of elements of the module $M$. So, distributive submodules can be also characterized in the following way; where we refer to $[9$, Theorem 1.6] or [1, Proposition 1.1] for condition (b), and to [3] for condition (c).

Proposition 3.1. Let $M$ be a right $R$-module, and $N \subseteq M$ be a submodule, the following statements are equivalent:

(a) $N \subseteq M$ is a distributive submodule.

(b) $(N: m)+(m R: n)=R$ for any $m \in M$ and $n \in N$.

(c) For every submodule $H \subseteq M$ the modules $N /(N \cap H)$ and $H /(N \cap H)$ have no non-zero isomorphic subfactors.

(d) For every submodule $H \subseteq M$, the modules $N /(N \cap H)$ and $H /(N \cap H)$ have no simple isomorphic subfactors.

(e) For any $m \in M$ and $n \in N$, the cyclic modules $(n+(N \cap m R)) R$ and $m R /(N \cap$ $m R)$ have no non-zero isomorphic subfactors.

(f) For any $m \in M$ and $n \in N$, the cyclic modules $(n+(N \cap m R)) R$ and $m R /(N \cap$ $m R$ ) have no simple isomorphic subfactors.

Proof. (a) $\Rightarrow$ (b) By hypothesis we have $N \cap((n-m) R+m R)=(N \cap(n-m) R)+$ $(N \cap m R)$; hence

$$
n=x+y, \text { where } x \in N \cap(n-m) R \text { and } y \in N \cap m R .
$$

Let $a, b \in R$ such that $x=(n-m) a$, hence $m a=n a-x \in N$, and $y=m b$. In addition, we have $n(1-a)=n-n a=x+y-n a=(n-m) a+m b-n a=m(b-a) \in$ $m R$. As a consequence, $(N: m)+(m R: n)=R$. 
(b) $\Rightarrow$ (a) For any $X, Y \subseteq M$ we always have $(N \cap X)+(N \cap Y) \subseteq N \cap(X+Y)$. On the other hand, let $n=x+y \in N \cap(X+Y)$, where $x \in X$ and $y \in Y$, and consider the pair $n \in N$ and $x \in M$. By hypothesis, we have $(N: x)+(x R$ : $n)=R$, there exist $a \in(N: x), b \in(x R: n)$ such that $a+b=1$, and we have: $n=x a+y a+n b$; since $x a, n b \in N \cap X$, hence $y a=n-x a-n b \in N$, whence ya $\in N \cap Y$. Therefore, $n \in(N \cap X)+(N \cap Y)$.

(b) $\Rightarrow$ (c) For any non-zero subfactor $S F_{1}$ of $N / N \cap H$, and any non-zero subfactor $S F_{2}$ of $H /(N \cap H)$, let us consider the diagram

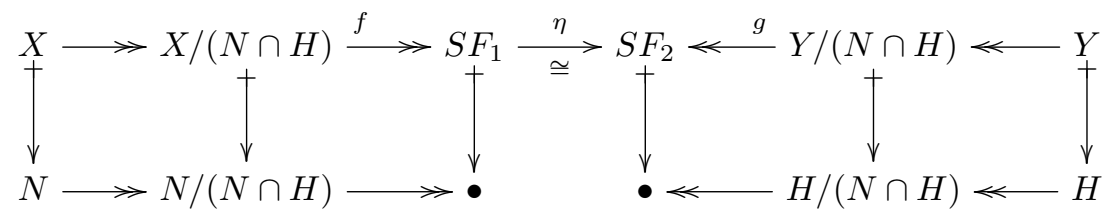

there exists $0 \neq x \in X$ such that $f(\bar{x}) \neq 0$, where $\bar{x}=x+(N \cap H)$. Let $y \in Y$ such that $\eta f(\bar{x})=g(\bar{y})$, where $\bar{y}=y+(N \cap H)$. By the hypothesis $(N: y)+(y R: x)=R$, if $1=a+b$ with $a \in(N: y), b \in(y R: x)$, then $x=x(a+b)=x a+x b$. On the other hand $\eta f(\overline{x a})=g(\bar{y} a)=0$, whence $\overline{x a} \in \operatorname{Ker}(f)$; since $\overline{x b} \in \operatorname{Ker}(f)$, we have $\bar{x} \in \operatorname{Ker}(f)$, which is a contradiction.

(c) $\Rightarrow$ (d), (e) $\Rightarrow$ (f) They are trivial.

(f) $\Rightarrow$ (b) Let $x \in M$ and $n \in N$, if $(N: x)+(x R: n) \neq R$, there exists a maximal right ideal $\mathfrak{m} \subseteq R$ such that $(N: x)+(x R: n) \subseteq \mathfrak{m}$, and for any $a \in \mathfrak{m}$ we have $1-a \notin \mathfrak{m}$, hence $1-a \notin(N: x),(x R, n)$. We proceed as follows:

(1) Since $1-a \notin(N: x)$, then $(1-a) x \notin N \cap x R$, and for any $a \in \mathfrak{m}$ we have $\bar{x} \neq \bar{x} a$ in $M /(N \cap x R)$, i. e., $\bar{x} \mathfrak{m} \varsubsetneqq \bar{x} R$, and $x R /(N \cap x R)$ has a simple subfactor $\bar{x} R / \bar{x} \mathfrak{m} \cong R / \mathfrak{m}$.

(2) Since $1-a \notin(x R: n)$, then $n(1-a) \notin N \cap x R$, and for any $a \in \mathfrak{m}$ we have $\bar{n} \neq \bar{n} a$ in $M /(N \cap x R)$, i. e., $\bar{n} \mathfrak{m} \varsubsetneqq \bar{n} R$, and $\bar{n} R$ has a simple subfactor $\bar{n} R / \bar{n} \mathfrak{m} \cong R / \mathfrak{m}$.

In any case we have a contradiction.

As a consequence, of the above proposition, if $\operatorname{Mod}-R$ has only, up to isomorphism, one simple right $R$-module, for instance if either $R$ has only one maximal right ideal, i.e., $R$ is a local ring, then we have the following proposition; compare with [1].

Proposition 3.2. Let $R$ be a ring such that $\mathbf{M o d}-R$ has, up to isomorphism, only a simple right module, for any proper submodule $N \varsubsetneqq M$ the following statements are equivalent: 
(a) $N \subseteq M$ is distributive.

(b) $N \subseteq m R$ for any $m \in M \backslash N$.

(c) $N$ is comparable with every non-zero submodule of $M$.

In particular, if $0 \neq N \varsubsetneqq M$ is a distributive submodule, then $\operatorname{Soc}(M) \subseteq N$ and it is essential in $M$.

Proof. (a) $\Rightarrow$ (b) If $N \subseteq M$ is distributive and $m \in M \backslash N$ then $\frac{N}{N \cap m R}$ and $\frac{m R}{N \cap m R}$ have no simple isomorphic subfactors, hence one of them is equal to zero. If $\frac{m R}{N \cap m R}=0$, then $m R \subseteq N$, which is a contradiction, hence $\frac{N}{N \cap m R}=0$, and $N \subseteq m R$.

(b) $\Rightarrow$ (c) Let $H \subseteq M$ be a submodule. If $H \nsubseteq N$, there exists $h \in H \backslash N$, hence $N \subseteq h R \subseteq H$.

(c) $\Rightarrow$ (a) Let $H \subseteq M$ be a submodule, then either $N \subseteq H$, hence $\frac{N}{N \cap H}=0$, or $H \subseteq N$, hence $\frac{H}{N \cap H}=0$.

By (b) we have that $N \subseteq M$ is essential. If $H \subseteq M$ is simple and $H \nsubseteq N$, there exists $h \in H \backslash N$, and $N \subseteq h R \subseteq H$, so $N=H$, which is a contradiction. As a consequence, for any simple submodule $H \subseteq M$ we have $H \subseteq N$, and $\operatorname{Soc}(M) \subseteq$ $N$.

A second consequence of the afore-mentioned characterization of distributive submodules given in Proposition (3.1) is the following proposition.

Proposition 3.3. Let $M$ be a right $R$-module, $N \subseteq M$ a distributive submodule and $H \subseteq M$ a submodule such that $N \cap H=0$, then $\operatorname{Hom}_{R}(N, H)=0=$ $\operatorname{Hom}_{R}(H, N)$.

Proof. For any homomorphism $f: N \longrightarrow H$ we have $\operatorname{Im}(f)$ is a common subfactor of $N$ and $H$, hence $\operatorname{Im}(f)=0$, and $f=0$. The same happens for any homomorphism $g: H \longrightarrow N$.

Finally, we observe that distributive submodules are preserved by some module constructions.

Proposition 3.4. Let $M$ be a right $R$-module, the following statements hold:

(1) If $N \subseteq M$ is a distributive submodule, for any submodule $H \subseteq M$ the submodule $(N+H) / H \subseteq M / H$ is distributive.

(2) For every family of distributive submodules $\left\{N_{i} \subseteq M \mid i \in I\right\}$ the sum $\sum_{i} N_{i} \subseteq$ $M$ is a distributive submodule. 
(3) If $N_{1}, N_{2} \subseteq M$ are distributive submodules, then $N_{1} \cap N_{2} \subseteq M$ is distributive.

Proof. (1) Since $N \subseteq M$ is distributive, for any $m \in M$ and any $n \in N$ we have $(N: m)+(m R: n)=R$. The result follows from the following inclusions

$$
(N: m) \subseteq\left(\frac{N+H}{H}: \bar{m}\right) \text { and }(m R: n) \subseteq(\bar{m} R: \bar{n}),
$$

where $\bar{x}=x+H$ for any $x \in M$.

(2) and (3) They are well known for finite join and meet of distributive elements of a lattice. It is not difficult to see that in the case of sum it can be extended to the infinite case.

If $A$ is a commutative ring and $\Sigma \subseteq A$ a multiplicatively closed subset, then we have:

Proposition 3.5. Let $M$ be an $A$-module; if $N \subseteq M$ is distributive, then $\Sigma^{-1} N \subseteq$ $\Sigma^{-1} M$ is distributive.

Proof. We apply Proposition (3.1(b)). Let $\frac{m}{1} \in \Sigma^{-1} M$, and $\frac{n}{1} \in \Sigma^{-1} N$. We have the equality:

$\left(\Sigma^{-1} N: \frac{m}{1}\right)=\left\{\frac{a}{s} \in \Sigma^{-1} A \mid\right.$ exists $t \in \Sigma$, such that mat $\left.\in N\right\}=\Sigma^{-1}(N: m)$, and $\left(\frac{m}{1} \Sigma^{-1} A: \frac{n}{1}\right)=\Sigma^{-1}(m A: n)$.

Since $(N: m)+(m A: n)=A$, then $\left(\Sigma^{-1} N: \frac{m}{1}\right)+\left(\frac{m}{1} \Sigma^{-1} A: \frac{n}{1}\right)=\Sigma^{-1} A$, and $\Sigma^{-1} N \subseteq \Sigma^{-1} M$ is distributive.

In particular, if $\mathfrak{p} \subseteq A$ is a prime ideal, and consider $\Sigma=A \backslash \mathfrak{p}$, then we have

Corollary 3.6. Let $M$ be an $A$-module and $\mathfrak{p} \subseteq A$ be a prime ideal. If $N \subseteq M$ is distributive, then $N_{\mathfrak{p}} \subseteq M_{\mathfrak{p}}$ is distributive.

Let us consider the following example.

Example 3.7. Let $M=\mathbb{Z}_{8}$ the cyclic abelian group of eight elements. Since $\mathcal{L}\left(\mathbb{Z}_{8}\right)$ is a distributive lattice, then every submodule is distributive, hence $\operatorname{Soc}\left(\mathbb{Z}_{8}\right)=$ $4 \mathbb{Z}_{8} \varsubsetneqq 2 \mathbb{Z}_{8}$ are proper distributive submodules. Otherwise, $\mathbb{Z}_{8}$ has no nontrivial direct summands, hence $\mathcal{L}\left(\mathbb{Z}_{8}\right)$ has no a lattice decomposition, see next section.

This means that the existence of distributive submodules does not imply a lattice decomposition. On the other hand, for every lattice decomposition $M=M_{1} \oplus M_{2}$ we shall prove that $M_{1}$ and $M_{2}$ are distributive submodules. 


\section{Complemented distributive submodules}

Let $M=N \oplus H$ be a decomposition, and let us denote $i_{1}: N \longrightarrow M$ and $i_{2}: H \longrightarrow M$ the inclusions and $q_{1}: M \longrightarrow N, q_{2}: M \longrightarrow H$ the projections. If $p_{1}: M \longrightarrow M / N$ is the projection, there is an isomorphism $f: M / N \cong H$ such that $f p_{1}=q_{2}$; thus, we have a diagram involving the lattices:

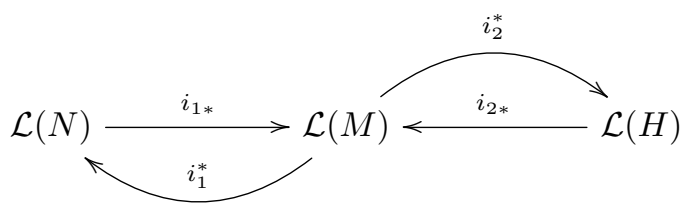

Being $i_{1 *}$ and $i_{2 *}$ lattice homomorphisms. On the other hand, $i_{1}^{*}$ and $i_{2}^{*}$ are $\wedge^{-}$ homomorphisms, i.e., $i_{j}^{*}\left(Y_{1} \wedge Y_{2}\right)=i_{j}^{*}\left(Y_{1}\right) \wedge i_{j}^{*}\left(Y_{2}\right)$, for every $Y_{1}, Y_{2} \subseteq M, j=$ 1,2 , and they are lattice homomorphisms whenever $N$, or equivalently $H$, is a distributive submodule. In this case, $\mathcal{L}(M)$ is the direct product of $\mathcal{L}(N)$ and $\mathcal{L}(H)$; and for every $Y \subseteq M$ we have $Y=i_{1 *} i_{1}^{*}(Y) \vee i_{2 *} i_{2}^{*}(Y)$.

The first result is a direct consequence of the characterizations of distributive submodules, in Proposition (3.1), which are direct summands, i.e., complemented distributive submodules.

Lemma 4.1. Let $M=N \oplus H$ be a direct sum, the following statements are equivalent:

(a) $N \subseteq M$ is distributive.

(b) $N$ and $H$ have no isomorphic simple subfactors.

(c) $\operatorname{Ann}(n)+\operatorname{Ann}(h)=R$ for any $n \in N$ and $h \in H$.

(d) For any submodule $X \subseteq M$ we have $X=(X \cap N)+(X \cap H)=(X+N) \cap(X+H)$.

(e) $H \subseteq M$ is distributive.

If $M$ is a right $R$-module satisfying the equivalent statements in the above lemma we say that $M=N \oplus H$ is a lattice decomposition of $M$.

In this context, if $A$ is a commutative ring, we have the following result that characterizes complemented distributive submodules.

Corollary 4.2. Let $A$ be a commutative ring, $M$ an $A$-module, and $M=N \oplus H$ be a decomposition in a direct sum, the following statements are equivalent:

(a) $N \subseteq M$ is a distributive submodule.

(b) $\operatorname{Supp}(N) \cap \operatorname{Supp}(H)=\varnothing$.

(c) $H \subseteq M$ is a distributive submodule. 
Proof. (a) $\Rightarrow$ (b) By Lemma (4.1), for any $n \in N$ and any $h \in H$ we have $\operatorname{Ann}(n)+\operatorname{Ann}(h)=A$. If $\mathfrak{p} \in \operatorname{Supp}(N) \cap \operatorname{Supp}(H)$, there exist $n \in N$ and $h \in H$ such that $(R n)_{\mathfrak{p}} \neq 0$, and $(R h)_{\mathfrak{p}} \neq 0$, hence $\operatorname{Ann}(n) \subseteq \mathfrak{p}$ and $\operatorname{Ann}(h) \subseteq \mathfrak{p}$, which is a contradiction.

(b) $\Rightarrow$ (a) If $N$ is not distributive, by Lemma (4.1), $N$ and $H$ have isomorphic simple subfactors, hence $\operatorname{Supp}(N) \cap \operatorname{Supp}(H) \neq \varnothing$, which is a contradiction.

As a consequence, if in addition $A$ is a noetherian ring, then lattice decomposition is inherited by injective hulls.

Corollary 4.3. Let $A$ be a commutative noetherian ring, $M$ an $A$-module, and $M=N \oplus H$ be a lattice decomposition, then $E(M)=E(N) \oplus E(H)$ is a lattice decomposition.

Proof. It is a direct consequence of the well known fact that for any $A$-module $N$ we have $\operatorname{Supp}(N)=\operatorname{Supp}(E(N))$.

Also we have the following straightforward result.

Lemma 4.4. Let $M$ be a right $R$-module such that $\mathcal{L}(M)$ is a direct product of two lattices, say $\mathcal{L}(M)=\mathcal{L}_{1} \times \mathcal{L}_{2}$, there exist $M_{1}, M_{2} \subseteq M$ such that

(1) $M=M_{1} \oplus M_{2}$.

(2) $M_{1}$ and $M_{2}$ are distributive submodules.

(3) $\mathcal{L}_{i} \cong \mathcal{L}\left(M_{i}\right)$, for every $i=1,2$.

(4) $\mathcal{L}(M)=\left[0, M_{1}\right] \times\left[0, M_{2}\right]$.

(5) There exists an idempotent endomorphism $e \in \operatorname{End}_{R}(M)$ such that $e(M)=M_{1}$, and $(1-e)(M)=M_{2}$. In addition, $e_{\mid M_{1}}=i d_{M_{1}}$, and $(1-e)_{\mid M_{2}}=i d_{M_{2}}$.

The existence of a non trivial idempotent endomorphism in $\operatorname{End}_{R}(M)$ is necessary, but it is not sufficient to get a lattice decomposition, i. e., not every idempotent endomorphism $e \in \operatorname{End}_{R}(M)$ defines a lattice decomposition of $\mathcal{L}(M)$. Let us illustrate it by some examples.

Example 4.5. Let us consider the abelian group $M=\mathbb{Z}_{2} \times \mathbb{Z}_{2}$. It is clear that $M$ has not non trivial distributive submodules, but $\operatorname{End}(M)$ has non trivial idempotents. Indeed, the ring $\operatorname{End}\left(\mathbb{Z}_{2} \times \mathbb{Z}_{2}\right)=M_{2}\left(\mathbb{Z}_{2}\right)$ has six non trivial idempotent endomorphisms $\left(\begin{array}{ll}1 & 1 \\ 0 & 0\end{array}\right),\left(\begin{array}{ll}1 & 0 \\ 1 & 0\end{array}\right),\left(\begin{array}{ll}0 & 0 \\ 1 & 1\end{array}\right),\left(\begin{array}{ll}0 & 1 \\ 0 & 1\end{array}\right),\left(\begin{array}{ll}1 & 0 \\ 0 & 0\end{array}\right)$ and $\left(\begin{array}{ll}0 & 0 \\ 0 & 1\end{array}\right)$, but no one of them defines a lattice decomposition. 
Example 4.6. In the positive we have: If we consider the abelian group $M=$ $\mathbb{Z}_{2} \times \mathbb{Z}_{3}$, then $\operatorname{End}\left(\mathbb{Z}_{2} \times \mathbb{Z}_{3}\right) \cong \operatorname{End}\left(\mathbb{Z}_{2}\right) \times \operatorname{End}\left(\mathbb{Z}_{3}\right)$; this ring decomposes, and there is a non trivial idempotent that produces a lattice decomposition of $M$.

The next example shows that in a lattice decomposable module not every idempotent endomorphism provides a lattice decomposition.

Example 4.7. Let us consider the abelian group $M=\mathbb{Z}_{2} \times \mathbb{Z}_{2} \times \mathbb{Z}_{3}$. The lattice of all subgroups is:

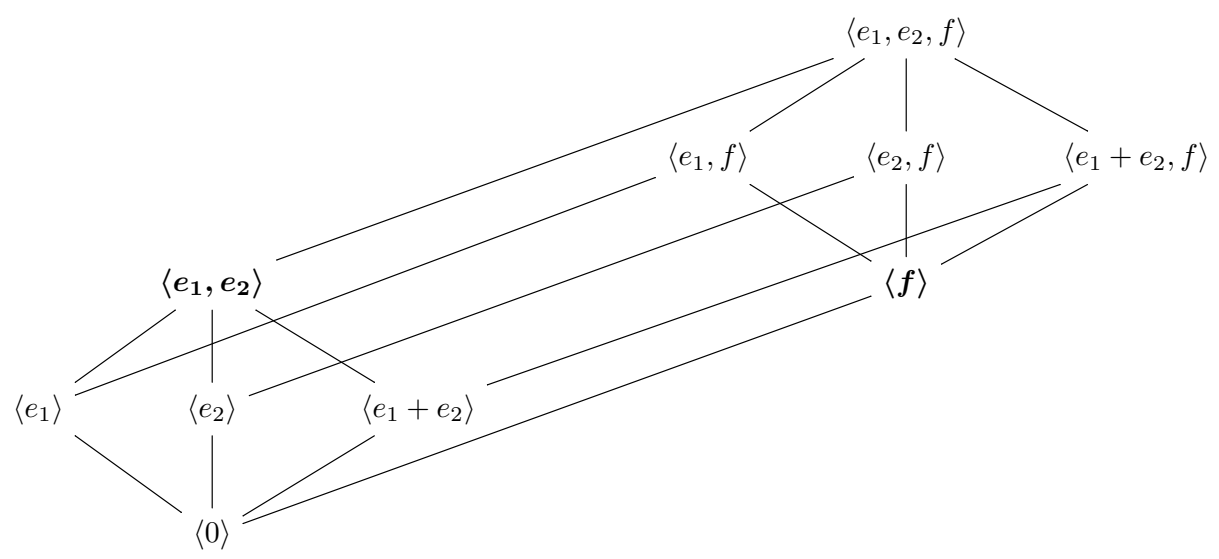

The decomposition given by $N_{1}=\left\langle e_{1}, e_{2}\right\rangle, N_{2}=\langle f\rangle$ corresponds to the idempotent central endomorphism $e \in \operatorname{End}(M)$ defined by

$$
e\left\{\begin{array}{l}
\left(e_{1}\right)=e_{1} \\
\left(e_{2}\right)=e_{2} \\
(f)=0
\end{array} \quad 1-e\left\{\begin{array}{l}
\left(e_{1}\right)=0 \\
\left(e_{2}\right)=0 \\
(f)=f
\end{array}\right.\right.
$$

and it defines the lattice decomposition of $\mathcal{L}(M)$ represented in the above diagram. In addition, $e$ defines a lattice decomposition of the $\operatorname{ring} S=\operatorname{End}_{R}(M)$.

Question 4.8. Does every central idempotent endomorphism in $\operatorname{End}_{R}(M)$ induce a lattice decomposition of $\mathcal{L}(M)$ ?

The answer is no, as the following example shows.

Example 4.9. Let us consider $M=\mathbb{Z}_{(2)} \times \mathbb{Z}_{(3)}$, where $\mathbb{Z}_{(2)}$ and $\mathbb{Z}_{(3)}$ are the localization of $\mathbb{Z}$ at $2 \mathbb{Z}$ and $3 \mathbb{Z}$, respectively. We claim $\operatorname{Hom}\left(\mathbb{Z}_{(2)}, \mathbb{Z}_{(3)}\right)=0$. Indeed, for any $f \in \operatorname{Hom}\left(\mathbb{Z}_{(2)}, \mathbb{Z}_{(3)}\right)$, let $f(1)=\frac{a}{d}$, then $f\left(\frac{1}{3}\right)=\frac{b}{c}$ and it satisfies: $3 \frac{b}{c}=\frac{a}{d}$, and $3 b d=a c$. By hypothesis $3 \nmid c$, hence $3 \mid a$. Similarly, if $f\left(\frac{1}{3^{t}}\right)=\frac{b}{c}$, then $3^{t} d b=a c$, and $3^{t} \mid a$ for every $t \in \mathbb{N}$, which implies $a=0$. 
It is clear that $\operatorname{End}(M)=\operatorname{End}\left(\mathbb{Z}_{(2)}\right) \times \operatorname{End}\left(\mathbb{Z}_{(3)}\right)$; hence, in $\operatorname{End}(M)$ there exist central idempotent elements. Since $\mathbb{Z}_{(2)}$ and $\mathbb{Z}_{(3)}$ have a non zero isomorphic submodule in common, then $\mathbb{Z}_{(2)} \subseteq M$ is not distributive.

Remark 4.10. Observe that any central idempotent element $e \in \operatorname{End}_{R}(M)=S$ induces a complemented distributive two-sided ideal $e S \subseteq S$, with complement $(1-e) S$, see Corollary (4.12) below. The above example shows that if $e \in \operatorname{End}_{R}(M)$ is a central idempotent element, then the submodule $e(M) \subseteq M$ is not necessarily distributive, even if $S$ has a lattice decomposition.

In conclusion, the question is: how we may describe the idempotent elements in $\operatorname{End}_{R}(M)$ that produce lattice decomposition? The next theorem provides the answer.

Theorem 4.11. Let $M$ be a right $R$-module, $N \subseteq M$ be a direct summand, and let $e \in \operatorname{End}_{R}(M)$ be an idempotent such that $e(M)=N$, the following statements are equivalent:

(a) $N$ is complemented distributive.

(b) $e$ is a central and $e(X) \subseteq X$ for any submodule $X \subseteq M$.

Proof. If $N \subseteq M$ is a complemented distributive submodule with complement $H$, then for every submodule $X \subseteq M$ we have $X=(N \cap X)+(H \cap X)=(e(M) \cap$ $X)+((1-e)(M) \cap X)$, that expressed in terms of the endomorphism $e$, implies:

$$
e(X)=e(e(M) \cap X)+e((1-e)(M) \cap X) \subseteq e(M) \cap X \subseteq X,
$$

Hence a necessary condition on the endomorphism $e$ to get a complemented distributive submodule is $e(X) \subseteq X$, for any submodule $X \subseteq M$. This is also a sufficient condition; indeed, if $e(X) \subseteq X$ (or equivalently $(1-e)(X) \subseteq X)$, then, for any element $x \in X$ we have $x=e(x)+(1-e)(x)$, where $e(x) \in e(M) \cap X$ and $(1-e)(x) \in(1-e)(M) \cap X$.

Let $M$ be a right $R$-module $M$, for any complemented distributive submodule $N \subseteq M$ with complement $H$, following Cohn's theory in [2], for any homomorphism $f: N \longrightarrow H$ we define a submodule $\Gamma(f)=\{(x, f(x)) \in M \mid x \in N\}$. Since $\Gamma(f)$ is a complement of $N$, and it is unique, it follows that $\Gamma(f)=\Gamma(0)=N$. In particular, $\operatorname{End}_{R}(N, H)=0$, hence we have $\operatorname{End}_{R}(M) \cong \operatorname{End}_{R}(N) \times \operatorname{End}_{R}(H)$. As a consequence $\operatorname{End}_{R}(N)$ is a direct summand ideal of $\operatorname{End}_{R}(M)$, and there exists a central idempotent $e \in \operatorname{End}_{R}(M)$ such that $\operatorname{End}_{R}(N)=e \operatorname{End}_{R}(M)$. Therefore, the idempotent endomorphism that defines the lattice decomposition is central. 
An idempotent endomorphism $e \in \operatorname{End}_{R}(M)$ is named fully invariant if $e(X) \subseteq$ $X$ for any submodule $X \subseteq M$.

Corollary 4.12. Let $R$ be a ring, and $e \in R$ idempotent, the following statements are equivalent:

(a) $e R \subseteq R$ is a complemented distributive right ideal.

(b) $e$ is central.

(c) $R=R e \times R(1-e)$ is a direct product of rings.

In this case $R$ has a lattice decomposition in a direct product of two (twosided) ideals: Re and $R(1-e)$, and conversely; any direct sum decomposition $R=\mathfrak{A} \oplus \mathfrak{B}$, with two (two-sided) ideals, is a lattice decomposition.

Remark 4.13. Observe that if $N \subseteq M$ is a complemented distributive submodule, defined by the idempotent endomorphism $e \in \operatorname{End}_{R}(M)$, then $\operatorname{End}_{R}(M) e \subseteq$ $\operatorname{End}_{R}(M)$ is a complemented distributive two-sided ideal; therefore a ring with unity $e$.

In particular, we have the next result that enhances [9, Proposition 4.3] and [1, Proposition 1.3].

Lemma 4.14. Every complemented distributive submodule $N \subseteq M$ is stable under any $f \in \operatorname{End}_{R}(M)$.

Proof. By hypothesis there exists $e \in \operatorname{End}_{R}(M)$, central idempotent, such that $N=e(M)$. For every endomorphism $f \in \operatorname{End}_{R}(M)$ we have $f(N)=f e(M)=$ ef $(M) \subseteq e(M)=N$.

When we analysed the question if every central idempotent endomorphism $e \in$ $\operatorname{End}_{R}(M)$ defines a complemented distributive submodule $e(M) \subseteq M$. We found that Example (4.9) gave a negative answer. A necessary and sufficient condition in order to get $e(M) \subseteq M$ a complemented distributive submodule, as we have seen before, is that for every submodule $X \subseteq M$ we have $e(X) \subseteq X$. In consequence, for every element $m \in M$ we have $e\langle m\rangle \subseteq\langle m\rangle$, and there exists $a_{m} \in R$ such that $e m=m a_{m}$.

Let us explore this condition in order to get more examples of complemented distributive submodules.

Of particular interest is the situation in which $A$ is a commutative ring. In this case we have:

Proposition 4.15. Let $A$ be a commutative ring and $N \subseteq M$ be a submodule of the $A$-module $M$, the following statements are equivalent: 
(a) $N \subseteq M$ is a complemented distributive submodule.

(b) There exists a central idempotent endomorphism e $\in \operatorname{End}_{A}(M)$ with $e(M)=N$ and e belongs to the closure of $A / A n n(M) \subseteq \operatorname{End}_{A}(M)$ in the finite topology, i.e., the topology with subbase of neighbourhood of any $f \in \operatorname{End}_{A}(M)$ given by $B\left(\left\{m_{1}, \ldots, m_{t}\right\}, f\right)=\left\{g \in \operatorname{End}_{A}(M) \mid g\left(m_{i}\right)=f\left(m_{i}\right), i=1, \ldots, t\right\}$.

Proof. (a) $\Rightarrow$ (b) Let $N \subseteq M$ be a complemented distributive submodule and $e \in \operatorname{End}_{A}(M)$ be a central idempotent element such that $e(M)=N$, and $e(X) \subseteq X$ for every submodule $X \subseteq M$. If $H=(1-e)(M)$, for any $m \in M$ there are $n \in N$ and $h \in H$ such that $m=n+h$, and there exists $a \in A$ such that $n=e(m)=m a=(n+h) a$, hence $h a=0$ and $n(1-a)=0$.

Let $m_{1}, \ldots, m_{t} \in M$ with $m_{i}=n_{i}+h_{i}$ and $e\left(m_{i}\right)=m_{i} a_{i}$. Let us consider new elements: $m_{i j}=n_{i}+h_{j}$; observe that there are elements $a_{i j} \in A$ such that $e\left(m_{i j}\right)=m_{i j} a_{i j}$ and $a_{i i}=a_{i}$; therefore $n_{i j}\left(1-a_{i j}\right)=0$ and $h_{i j} a_{i j}=0$, being $n_{i j}=n_{i}$ and $h_{i j}=h_{j}$, for any $i, j \in\{1, \ldots, t\}$.

If we fix $i$, we have the elements $m_{i 1}=n_{i}+h_{1}, \ldots, m_{i t}=n_{i}+h_{t}$, that satisfy:

$$
\begin{aligned}
& n_{i}-n_{i}\left(a_{i 1} \cdots a_{i t}\right)=n_{i}\left(1-a_{i 1} \cdots a_{i t}\right)=0 \\
& h_{j}\left(a_{i 1} \cdots a_{i t}\right)=0 \\
& e\left(m_{i j}\right)=m_{i j}\left(a_{i 1} \cdots a_{i t}\right)
\end{aligned}
$$

Thus we may assume $a_{i 1}=\cdots=a_{i t}$, and all of them are equal to the product $a_{i 1} \cdots a_{i t}$ for the former $a_{i j}$. How this can be done for every index $i$, then we may assume $a_{i 1}=\cdots=a_{i t}$ for every index $i$.

In this new context, if we fix $j$, we have elements $m_{1 j}=n_{1}+h_{j}, \ldots, m_{t j}=n_{t}+h_{j}$, that satisfy:

$$
\begin{aligned}
& n_{i}\left(1-a_{1 j}\right) \cdots\left(1-a_{t j}\right)=0, \text { for every index } i, \\
& \left(1-a_{1 j}\right) \cdots\left(1-a_{t j}\right)=1-\sum_{i} a_{i j}+\sum_{i_{1}<i_{2}} a_{i_{1} j} a_{i_{2} j}+\cdots+(-1)^{t} a_{1 j} \cdots a_{t j}, \\
& \text { let us denote } x=\sum_{i} a_{i j}-\sum_{i_{1}<i_{2}} a_{i_{1} j} a_{i_{2} j}+\cdots+(-1)^{t+1} a_{1 j} \cdots a_{t j}, \\
& h_{j} x=0, \\
& e\left(m_{i j}\right)=m_{i j} x .
\end{aligned}
$$

Thus we may assume $a_{1 j}=\cdots=a_{t j}$, and all of them are equal to the element $x$ defined just before using the former $a_{i j}$. how this can be done for every index $j$, then we may assume $a_{1 j}=\cdots=a_{t j}=x$ for every index $j$. In consequence we have found that $e\left(m_{i j}\right)=m_{i j} x$, and in particular $x \in B\left(\left\{m_{1}, \ldots, m_{t}\right\}, e\right) \cap A$. Therefore, $e$ belongs to the closure of $A / \operatorname{Ann}(M)$ in the finite topology of $\operatorname{End}_{A}(M)$.

(b) $\Rightarrow$ (a) It is consequence of Theorem (4.11). 
Corollary 4.16. For any commutative ring $A$ and any finitely generated $A$-module $M$, if $N \subseteq M$ is a complemented distributive submodule with idempotent endomorphism e, there exists $a \in A$ such that $e(m)=$ ma for any $m \in M$.

Even in this particular case we may characterize complemented distributive submodules.

Corollary 4.17. Let $A$ be a commutative ring and let $M$ be a finitely generated A-module; every complemented distributive submodule $N \subseteq M$ determines an idempotent element in $A / A n n(M)$. And conversely, every non zero idempotent in $A / A n n(M)$ defines a nonzero complemented and distributive submodule of $M$.

Example 4.18. Let us consider the abelian group $M=\mathbb{Z}_{2} \times \mathbb{Z}_{2} \times Z_{3}$ in the Example (4.7); we have $\operatorname{Ann}(M)=6 \mathbb{Z}$, and the non trivial idempotents of $\mathbb{Z} / 6 \mathbb{Z}$ are $\overline{4}$ and $\overline{3}$, which define the subgroups $2 M=\langle f\rangle$ and $3 M=\left\langle e_{1}, e_{2}\right\rangle$.

\section{Decomposition of categories. Examples}

In this section we will apply the above result to study the decomposition of the category of right $R$-modules and the category $\sigma[M]$ defined by a right $R$-module $M$.

The category Mod $-R$. Each lattice decomposition of $R$, as right $R-$ module, is defined by a central idempotent element $e \in R$, hence $R=R e \times R(1-e)$, being $R e$ and $R(1-e)$ rings (and (twosided) ideals). Therefore, we have a decomposition of the module category as $\mathbf{M o d}-R \cong \mathbf{M o d}-e R \times \mathbf{M o d}-(1-e) R$.

The category $\sigma[M]$. The lattice decomposition theory of a right $R$-module $M$ is closely linked to the structure of the $M$ module and also to the structure of submodules of the modules it generates; there is a category that studies precisely these modules: the category $\sigma[M]$. The category $\sigma[M]$, as defined in [12], is the full subcategory of Mod $-R$ whose objects are all the right $R$-modules isomorphic to modules subgenerated by $M$, i.e., submodules of factors of direct sums of copies of $M$.

Our aim is to study under which circumstances the category $\sigma[M]$ is a direct product of two categories $\mathcal{N}$ and $\mathcal{H}$.

Let us assume $F: \sigma[M] \cong \mathcal{N} \times \mathcal{H}$, without losing of generality we may assume $\mathcal{N}$ and $\mathcal{H}$ are subcategories of $\sigma[M]$, the objects of $\mathcal{N} \times \mathcal{H}$ are pairs $(X, Y)$, where $X$ is and object of $\mathcal{N}$ and $Y$ an object of $\mathcal{H}$, and $F(M)=(N, H)$ satisfying $M=N \oplus H$.

First we need a technical result, which will be useful in later developments. 
Proposition 5.1. Let $N \subseteq M$ be a complemented distributive submodule, for every index set I we have $N^{(I)} \subseteq M^{(I)}$ is a complemented distributive submodule. The reciprocal also holds.

Proof. It is obvious that if $M=N \oplus H$, then $M^{(I)}=N^{(I)} \oplus H^{(I)}$. Otherwise, if $S$ is a simple subfactor of $N^{(I)}$, there exists a finite subset $F \subseteq I$ such that $S$ is a subfactor of $N^{(F)}$, hence $S$ is a subfactor of $N$. Since $N$ and $H$ have no isomorphic simple subfactors, $N^{(I)}$ y $H^{(I)}$ have no isomorphic simple subfactors, hence $N^{(I)} \subseteq M^{(I)}$ is a complemented distributive submodule.

Every submodule $Z \subseteq M$ corresponds to a pair $(X, Y)$, object of $\mathcal{N} \times \mathcal{H}$ satisfying $X \subseteq N, Y \subseteq H$ and $Z=X \oplus Y$, hence $N \subseteq M$ is a distributive submodule of $M$ with complement $H$.

Conversely, for any complemented and distributive submodule $N \subseteq M$, with complement $H \subseteq M$, and any index set $I$, we have $N^{(I)} \subseteq M^{(I)}$ is a complemented and distributive submodule with complement $H^{(I)}$, see Proposition (5.1), and any submodule $X \subseteq M^{(I)}$ can be written as $X=\left(X \cap N^{(I)}\right) \oplus\left(X \cap H^{(I)}\right)$, hence $\frac{M^{(I)}}{X} \cong$ $\frac{N^{(I)}}{X \cap N^{(I)}} \oplus \frac{H^{(I)}}{X \cap H^{(I)}} \cong \frac{N^{(I)}+X}{X} \oplus \frac{H^{(I)}+X}{X}$, and $\frac{N^{(I)}}{X \cap N^{(I)}} \cong \frac{N^{(I)}+X}{X} \subseteq \frac{M}{X}$ is a complemented and distributive submodule. As a consequence, every submodule $Y \subseteq \frac{M^{(I)}}{X}$ can be written as $Y=\left(Y \cap \frac{N^{(I)}+X}{X}\right) \oplus\left(Y \cap \frac{N^{(I)}+X}{X}\right)$, where $Y \cap \frac{N^{(I)}+X}{X}$ is an object of $\sigma[N]$, and $Y \cap \frac{H^{(I)}+X}{X}$ is an object of $\sigma[H]$. Therefore there is a category isomorphism $\sigma[M] \cong \sigma[N] \times \sigma[H]$. Compare with $[11$, Proposition 2.2] and [13, 2.4].

Theorem 5.2. With the above assumptions. Let $M$ be a right $R$-module, the following statements are equivalent:

(a) $\sigma[M]$ is a direct product of two categories, $\sigma[M] \cong \sigma[N] \times \sigma[H]$.

(b) $M$ has a complemented and distributive submodule $N \subseteq M$, with complement $H$.

As a consequence of Corollary (4.3), if $A$ is a noetherian commutative ring, $M$ an $A$-module, and $E(M)$ its injective hull, for any complemented distributive submodule $N \subseteq M$ we have that $E(N) \subseteq E(M)$ is distributive. This result does not necessarily hold in a non-commutative framework as the following example shows, see [4]. 
Example 5.3. Let $K$ be a field and $R=\left(\begin{array}{ll}K & 0 \\ K & K\end{array}\right)$ be a ring. The maximal right ideals of $R$ are $\mathfrak{P}=\left(\begin{array}{ll}K & 0 \\ K & 0\end{array}\right)$ and $\mathfrak{Q}=\left(\begin{array}{cc}0 & 0 \\ K & K\end{array}\right)$; hence there are, up to isomorphism, two different simple right $R$-modules. Let us consider the right $R-$ module $N=\left(\begin{array}{cc}K & 0 \\ 0 & 0\end{array}\right)$, which is isomorphic to $R / \mathfrak{Q}$, and the cyclic right $R$-module $E=\left(\begin{array}{cc}K & K \\ 0 & 0\end{array}\right)$, generated by $\left(\begin{array}{ll}0 & 1 \\ 0 & 0\end{array}\right)$. Since $E$ is injective and the inclusion $N \subseteq E$ is essential, then $E$ is the injective hull of $N$. In addition we have an isomorphism $E / N \cong R / \mathfrak{P}$.

Consider now the right $R$-module $M=N \oplus(E / N)$. Since both factors are simple right $R$-module, $M$ has a lattice decomposition; $N \subseteq M$ is a complemented distributive submodule. Otherwise, $E(M)=E(N) \oplus E(E / N)=E \oplus(E / N)$, and $E \subseteq E(M)$ is not a distributive submodule. Indeed, $E(M)$ has no nontrivial complemented distributive submodules.

\section{References}

[1] A. Barnard, Distributive extensions of modules, J. Algebra, 70(2) (1981), 303315.

[2] P. M. Cohn, Free Rings and Their Relations, Second Edition, London Mathematical Society Monographs, 19, Academic Press, Inc., London, 1985.

[3] T. M. K. Davison, Distributive homomorphisms of rings and modules, J. Reine Angew. Math., 271 (1974), 28-34.

[4] J. M. Garcia, P. Jara and L. M. Merino, Decomposition of comodules, Comm. Algebra, 27(4) (1999), 1797-1805.

[5] G. Grätzer, General Lattice Theory, Birkhäuser Verlag, Basel-Stuttgart, 1978.

[6] C. U. Jensen, Arithmetical rings, Acta Math. Acad. Sci. Hungar., 17 (1966), 115-123.

[7] S. Rajaee, Multiplication modules on arithmetical rings, Int. J. Algebra, 7 (2013), 825-828.

[8] B. Stenström, Rings of Quotients, Springer-Verlag, Berlin, 1975.

[9] W. Stephenson, Modules whose lattice of submodules is distributive, Proc. London Math. Soc., 28(3) (1974), 291-310.

[10] A. A. Tuganbaev, Distributive extensions of modules, J. Math. Sci., 149(3) (2008), 1279-1285. 
[11] N. Vanaja, All finitely generated $M$-subgenerated modules are extending, Comm. Algebra, 24(2) (1996), 543-572.

[12] R. Wisbauer, Grundlagen der Modul- und Ringtheorie, Verlag Reinhard Fischer, Munich, 1988.

[13] R. Wisbauer, Decompositions of modules and comodules, Algebra and its Applications (Athens, OH, 1999), Contemp. Math., Amer. Math. Soc., Providence, RI, 259 (2000), 547-561.

\section{J. M. García}

Department of Applied Mathematics

University of Granada

E-18071 Granada, Spain

e-mail: jgarciah@ugr.es

P. Jara (Corresponding Author) and L. M. Merino

Department of Algebra and IAMAT-GR (Instituto de Matemáticas)

University of Granada

E-18071 Granada, Spain

e-mails: pjara@ugr.es (P. Jara)

lmerino@ugr.es (L. M. Merino) 